\title{
La aspergilosis pulmonar invasiva en la enfermedad pulmonar obstructiva crónica
}

\author{
JOSÉ MANUEL VALLE ${ }^{1}$, FRANCISCO JAVIER GONZÁLEZ-BARCALA², \\ JOSÉ MANUEL ÁLVAREZ-DOBAÑO ${ }^{1}$, LUIS VALDÉS C. ${ }^{1}$
}

'Servicio de NeumologíaComplexo Hospitalario de Santiago de Compostela. ${ }^{2}$ Servicio de NeumologíaComplexo Hospitalario de Pontevedra.

Recibido 16 marzo 2009 Aceptado 26 abril 2010. Correspondencia a: Luis Valdés Cuadrado Fax: +34-981583312 Teléfono:+34-607819141 E-mail: luis.valdes.cuadrado@sergas.es

\section{Invasive pulmonary aspergillosis in chronic obstructive pulmonary disease}

Invasive pulmonary aspergillosis (IPA) is a severe disease, specially among immunocompromised patients. Its frequency increases in other patients such as those with Chronic Obstructive Pulmonary Disease (COPD), mainly when steroids are prescribed. The most common form of presentation is a respiratory tract infection with poor response to antimicrobial treatment. The delay in its diagnosis is one of the main causes of its high lethality. Once suspected, respiratory secretion cultures, chest $X$ ray examination and computed tomography should be obtained and galactomannan, a marker of hematogenous dissemination of the microorganism, should be determined. Although the repeated isolation of Aspergillus spp is suggestive of invasive disease, the definitive diagnosis requires cytopathological confirmation. Further studies should be performed in these patients, since the available information was obtained from the observations made in immunocompromised patients, and may not be applicable accurately to API among COPD patients.

(Rev Med Chile 2010; 138: 612-620).

Key words: Aspergillosis; Chronic obstructive; Immunity; Pulmonary disease.
$\mathrm{E}$ 1 Aspergillus es un hongo que se encuentra ampliamente distribuido por todo el mundo. Entre sus numerosas especies, el Aspergillus fumigatus es el causante de, aproximadamente, $90 \%$ de las infecciones en humanos. El pulmón es el principal órgano afectado por el Aspergillus debido a la alta capacidad esporulativa de este hongo, y a que sus conidias son lo suficientemente pequeñas para poder alcanzar el alveolo ${ }^{1}$.

El Aspergillus spp puede causar varias enfermedades como son el aspergiloma, la aspergilosis broncopulmonar alérgica o la aspergilosis pulmonar invasiva (API) ${ }^{2}$.

La API en pacientes con enfermedad pulmonar obstructiva crónica (EPOC) es una enfermedad grave, con una tasa de mortalidad elevada, entre $91 \%$ y $95 \%{ }^{2,3}$. Parecen un factor relevante para este mal pronóstico las dificultades diagnósticas, derivadas de la inespecificidad de la presentación clínica y de la ausencia de pruebas complementarias no invasivas que puedan considerarse definitivas ${ }^{2,4}$. Además, en pacientes sin datos de inmunodepresión conocida, el diagnóstico puede retrasarse más por no plantearse inicialmente la posibilidad diagnóstica de $\mathrm{API}^{5}$.

A lo largo de las pasadas dos décadas se ha producido un incremento significativo de la incidencia de API, especialmente en pacientes gravemente inmunodeprimidos, como los tratados con quimioterapia o trasplantados. Pero en los últimos años también se ha visto, cada vez con más frecuencia, asociada a otras enfermedades, entre las cuales se incluye la $\mathrm{EPOC}^{2,3,6}$. Actualmente, entre $30 \%$ y $50 \%$ de casos de API se diagnostican en pacientes no neutropénicos, con una mortalidad similar a los neutropénicos ${ }^{4,7}$.

Este incremento de incidencia hace que, para algunos autores, la EPOC deba incluirse entre 


\title{
Tabla 1. Definiciones de aspergilosis pulmonar invasiva (API) en pacientes con enfermedad pulmonar obstructiva crónica (EPOC)
}

\begin{abstract}
API probada: Muestra histológica o citológica obtenida de una lesión pulmonar presente durante menos de 3 meses, en la que se observan hifas consistentes con Aspergillus y con evidencia de daño tisular, que se acompaña de alguno de los siguientes criterios:

1) Cultivo positivo de Aspergillus spp en una muestra de las vías aéreas inferiores

2) Test sérico de antígeno/anticuerpo positivo para Aspergillus Fumigatus (incluidas precipitinas)

3) Confirmación de que las hifas observadas son de Aspergillus por un método molecular, inmunológico y/o cultivo
\end{abstract}

API probable: Igual que la API probada pero sin la confirmación de que el Aspergillus es el responsable (los puntos 1, 2 y 3 están ausentes), o

Paciente EPOC grave (estadio III o IV de la GOLD), generalmente a tratamiento con corticoides, con aumento reciente de la disnea, radiografía o TC sugestivas ( $<3$ meses) y uno de los siguientes criterios:

1) Cultivo positivo y/o visualización microscópica de Aspergillus en las vías respiratorias inferiores

2) Anticuerpos en sangre positivos para Aspergillus (incluyendo precipitinas)

3) Dos tests consecutivos de galactomanano en sangre positivos

API posible: Paciente EPOC grave (estadio III o IV de la GOLD), generalmente a tratamiento con corticoides, con aumento reciente de la disnea, radiografía o TC sugestivas ( $<3$ meses) pero sin cultivo positivo, visualización microscópica del Aspergillus en muestras de la vía aérea inferior, o serología

Colonización: Paciente EPOC con cultivo positivo de Aspergillus en las vías aéreas inferiores sin aumento de la disnea, presencia de broncoespasmo, o de nuevos infiltrados pulmonares

GOLD: Global Initiative for Chronic Obstructive Lung Disease; TC: Tomografía Computarizada.

las patologías de base con alto riesgo de $\mathrm{API}^{8}$. La mayoría, aunque no todos, de los pacientes con EPOC que desarrollan API estaban en tratamiento con corticoides sistémicos ${ }^{2,3,8,9}$. Bulpa et al, en una revisión de varias series, encuentran que 43 de los 56 pacientes estudiados habían recibido corticoides en el momento del ingreso y, de éstos, 3 los habían tomado únicamente de forma inhalada ${ }^{2}$.

La falta de una definición consensuada, la ausencia de medidas de vigilancia de la infección, o la dificultad para diferenciar la colonización de la infección hacen que la incidencia de la API asociada a la EPOC sea difícil de valorar ${ }^{2}$.

Actualmente, el diagnóstico de API, tanto en la categoría de probada, probable o posible, está realizada en base a una población de pacientes inmunodeprimidos, fundamentalmente oncológicos y trasplantados, altamente susceptible a las infecciones fúngicas ${ }^{10,11}$. Recientemente se han propuesto los criterios de API posible, probable y probada en pacientes con EPOC (Tabla 1)2.

\section{Fisiopatología}

Las diferentes formas clínicas derivadas de la presencia de Aspergillus spp en la vía aérea deben verse como un proceso evolutivo, que se inicia en la colonización de la vía aérea, continúa con la ruptura de la barrera epitelial por las hifas del hongo (evento inicial de la invasión tisular), progresando a API, pudiendo posteriormente diseminarse al resto del organismo ${ }^{7}$.

No se conoce de forma definitiva el motivo por el cual algunos pacientes con EPOC desarrollan API, pero se han descrito diversos factores que parecen favorecer esta evolución (Tabla 2). El daño

\section{Tabla 2. Factores de riesgo de aspergilosis pulmonar invasiva}

- Exposición o colonización de la vía aérea por Aspergillus

- Gravedad de la enfermedad pulmonar de base

- Otra enfermedad grave

- Deterioro del aclaramiento muco-ciliar.

- Obras de construcción o renovación del hospital.

- Tratamiento corticoideo

- Respuesta anti-inflamatoria

- Otras infecciones

- Tratamiento con antibióticos.

- Factores genéticos. 
pulmonar será distinto según el estado inmunológico de los pacientes. En los neutropénicos se produce angioinvasión, hemorragia intraalveolar, e infartos pulmonares (Figura 1). En los no neutropénicos se producirá necrosis inflamatoria, pero sin invasión vascular, hemorragia o infarto ${ }^{12}$. Este modelo de lesión pulmonar no angioinvasiva también es el aspecto más característico de la API en modelos animales sometidos a tratamiento corticoideo $^{12}$.

La importancia de la exposición o colonización de la vía aérea por Aspergillus spp parece demostrada por diversos estudios. Así, en pacientes graves ingresados en Unidades de Cuidados Intensivos (UCI), entre los cuales se incluye $22 \%$ de EPOC, el aislamiento de este hongo en sus secreciones respiratorias se asocia en $28 \%$ de casos con $\mathrm{API}^{13}$. Otros autores observan, en un estudio también de UCI, con $44 \%$ de pacientes con EPOC, que la presencia de Aspergillus spp en secreciones respiratorias se asocia con mayor mortalidad ${ }^{14}$.

En el EPOC grave concurren diversos factores que deterioran la función ciliar, como el tabaquismo, los episodios repetidos de infección y el daño epitelial ${ }^{2,15}$. Esto puede facilitar la llegada del Aspergillus fumigatus a la capa epitelial y, desde ahí, invadirá, en primer lugar, la mucosa bronquial, a continuación el parénquima pulmonar adyacente $y$, finalmente, la vasculatura, lo que provocará infartos pulmonares ${ }^{16}$.

Los corticoides favorecen el crecimiento de algunos Aspergillus (especialmente fumigatus) ${ }^{17}$ disminuyendo la actividad antifúngica de los macrófagos alveolares ${ }^{18} \mathrm{e}$ inhibiendo a los neutrófilos ${ }^{19}$. Por otra parte, suprime la producción de citokinas Th- 1 y aumenta las Th- $2^{20}$. En cualquier caso, es poco frecuente que se produzcan infecciones graves relacionadas con el tratamiento corticoide con dosis inferiores a $10 \mathrm{mg} /$ día, o menos de $700 \mathrm{mg}$ acumulados ${ }^{21}$.

La respuesta inflamatoria es bifásica en procesos sépticos. La fase hiperinflamatoria inicial es seguida de un período de respuesta antiinflamatoria. Si esta reacción es excesiva puede condicionar un período de inmuno-parálisis, también conocido como síndrome de respuesta anti-inflamatoria compensatoria. En estos períodos se acentúa la vulnerabilidad del organismo a otras infecciones ${ }^{22,23}$.

Se ha descrito que tanto infecciones bacterianas como víricas pueden favorecer el desarrollo de

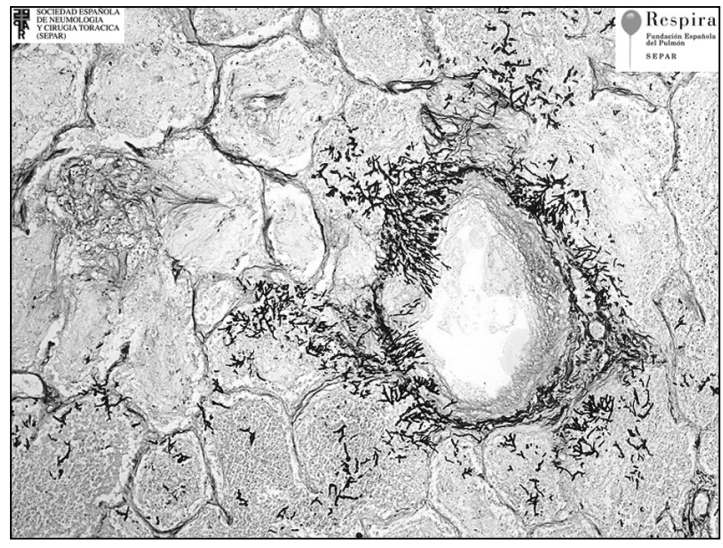

Figura 1. Hifas de aspergillus en los alvéolos, invadiendo la luz de estructuras vasculares.

$\mathrm{API}^{24,25}$. El mecanismo sería por depresión inmune transitoria y deterioro de la función ciliar, asociados a la infección precedente ${ }^{25}$.

Los pacientes tratados con antibióticos de amplio espectro presentan mayor riesgo de $\mathrm{API}^{13}$. Algunos posibles mecanismos serían porque estos tratamientos modifican la microbiota habitual, así como los patrones de resistencia y colonización por patógenos ${ }^{13}$.

También podrían influir factores genéticos. Se ha descrito, en modelos experimentales, que las proteínas del surfactante pulmonar pueden participar en la fagocitosis y eliminación de los Aspergillus ${ }^{26,27}$. También es conocida la diferente reactividad al Aspergillus en función de variantes genéticas de las proteínas del surfactante pulmonarr $^{28}$.

\section{Cuadro clínico}

En un paciente con EPOC, con un cuadro clínico sugestivo de infección respiratoria, e infiltrados pulmonares, que no responde al tratamiento antibiótico de amplio espectro, debe contemplarse la posibilidad de $\mathrm{API}^{2,3,9}$.

Predomina en varones con una edad media de 65 años. El tratamiento corticoideo sistémico está presente en $70 \%$ de los casos ya antes del ingreso, $y$ alcanza a $87 \%$ de los pacientes durante el ingreso hospitalario ${ }^{2}$.

Los síntomas de presentación son muy variables. Lo más frecuente es la disnea (32\%-48\% de 
casos), fiebre $(39 \%)$, tos $(26 \%)$, sibilancias $(8 \%$ $28 \%)$ o hemoptisis (12\%-15\%). Otros síntomas son menos frecuentes, como el incremento de expectoración o el dolor torácico ${ }^{2,3}$.

Con cierta frecuencia no suele sospecharse la aspergilosis, contemplándose otras posibilidades diagnósticas. Las hipótesis diagnósticas más frecuentemente establecidas al diagnóstico son la insuficiencia respiratoria aguda $(41 \%$ de los casos), EPOC agudizado (34\%), neumonía (20\%), e incluso shock séptico en un $5 \%$ de pacientes ${ }^{3}$.

El retraso entre el inicio de los síntomas y el diagnóstico (mediana de 8,5 días), puede ser una de las causas de la morbimortalidad de esta enfermedad ${ }^{2}$.

Los datos de la analítica sanguínea son inespecíficos. Suelen revelar leucocitosis, aumento de la proteína $\mathrm{C}$ reactiva, del fibrinógeno y de la lactato deshidrogenasa (LDH). También pueden presentar trombocitopenia leve ${ }^{2}$.

\section{Exploraciones complementarias en el diagnóstico de la API}

Ante la sospecha de infección respiratoria, se realizará cultivo de esputo. Su utilidad es limitada. La sensibilidad está alrededor de 50\%. Además puede haber otras especies de hongos, pero cuya respuesta al tratamiento es distinta ${ }^{2,3}$. Esta sensibilidad puede aumentar hasta $88 \%$ si se utilizan técnicas de fluorescencia ${ }^{29}$.

Aunque el Aspergillus spp pueda crecer en un medio de cultivo común, el crecimiento es mejor en medio específico para hongos. El cultivo positivo para Aspergillus spp no es diagnóstico per se, pero incrementará la sospecha de infección cuando el paciente no responde a antibióticos y, especialmente, en los pacientes tratados con esteroides. Los cultivos positivos repetidos son más sugestivos de infección ${ }^{30}$.

El cultivo de las secreciones respiratorias tiene un valor añadido al del diagnóstico clínico, ya que nos permitirá identificar la sensibilidad del germen a los fármacos disponibles ${ }^{31}$.

Cuando no se dispone de esputo, el material para cultivo se obtendrá por fibrobroncoscopia. La rentabilidad de los cultivos varía entre 46 y $77 \%{ }^{32,33}$, mientras que se demostraron rentabilidades más bajas en pacientes que recibían tratamiento antifúngico ${ }^{34}$. La utilidad diagnóstica de las muestras obtenidas por broncoscopia es superior en casos de neumonía prolongada o de lesiones pulmonares extensas. Brown et $\mathrm{al}^{35}$ sugieren que la positividad del lavado broncoalveolar (BAL) depende del tipo de API: 20\% en la forma angioinvasiva y $80 \%$ en la forma invasiva de la vía aérea. Curiosamente, el examen citológico parece ser más a menudo positivo que los cultivos ${ }^{36}$.

\section{Serología}

El antígeno galactomanano (GM) es un marcador de la diseminación hematógena del hongo, muy utilizado en pacientes neutropénicos, donde parece útil para establecer la sospecha de API antes de la aparición de la sintomatología. Su evaluación dos veces por semana permite ver la evolución de los niveles de galactomanano ${ }^{37}$. La antigenemia positiva precedió a la fiebre en 3,5 días, a los hallazgos en la radiografía simple de tórax en 8 días, a la TAC torácica en 6 días y a los resultados del primer cultivo de secreciones respiratorias en 9 días ${ }^{37}$. Para obtener estos resultados, las muestras de sangre deben tomarse al menos 2 veces por semana ${ }^{38}$. El GM también se puede detectar en el BAL, orina o líquido céfalo raquídeo ${ }^{3}$.

En pacientes no neutropénicos es más difícil de valorar. No está bien establecido el valor de una determinación puntual; y parece difícil su medición repetida en pacientes no neutropénicos. En pacientes no neutropénicos los resultados son contradictorios. Unos autores defienden su utilidad, con una sensibilidad de $88 \%$ y especificidad de $87 \% 39$; y por el contrario, otros destacan los falsos positivos relacionados con la colonización fúngica en el lavado broncoalveolar de los pacientes no inmunodeprimidos con infiltrados pulmonares ${ }^{40}$.

Globalmente el GM tiene una sensibilidad de $71 \%$ y una especificidad de $89 \%$, con un valor predictivo positivo entre $25 \%$ y $62 \%$, y un valor predictivo negativo entre $92 \%$ y $98 \%$, aunque con gran variabilidad entre los diferentes estu$\operatorname{dios}^{41,42}$. Estas discrepancias se deben, entre otras causas, al número de tests realizados por semana, la definición utilizada de API y el punto de corte elegido $^{37,41,42}$.

Una modificación en el proceso de las muestras de suero permite un incremento significativo de la sensibilidad del test de GM, además de acortar los tiempos de diagnóstico, aunque con una discreta disminución en la especificidad ${ }^{43}$. 
El GM también se ha aplicado en pacientes con EPOC gravemente enfermos y sospecha de API. En un estudio con 25 pacientes, 12 fueron positivos (40\%). Sin embargo, no se pueden extraer conclusiones definitivas debido a la pequeña muestra, el diseño retrospectivo del estudio y la ausencia de investigación sobre la existencia de factores confusores ${ }^{9}$. Una única determinación de GM podría ser útil para el diagnóstico de la API en pacientes no hematológicos ${ }^{44}$.

Un incremento en la antigenemia sobre un valor basal puede discriminar mejor que un simple valor por encima del normal. La elevación de la antigenemia durante la primera semana de observación estaba asociado con enfermedad progresiva, con una sensibilidad de $44 \%$, una especificidad de $87 \%$, y un valor predictivo positivo de $94 \%{ }^{44}$. Un aumento en la antigenemia también podría ser indicativo de fallo en el tratamiento $y$, por tanto, nos permitiría anticipar la decisión de modificar el tratamiento ${ }^{45}$.

El test del GM tiene varias limitaciones. Solamente un tercio de los pacientes tenían una respuesta antigénica positiva antes de que el diagnóstico de API se hiciera por TC. Tampoco se ha demostrado reducción de la mortalidad con la utilización de esta prueba. Se han descrito falsos positivos en pacientes que estaban recibiendo tratamiento con piperacilina/tazobactan o amoxicilina/clavulánico; así como reacciones cruzadas con otros hongos, bacterias, ciclofosfamida y comidas (vegetales enlatados, pasta, arroz). Por último, se desconoce si los resultados obtenidos en enfermedades hematológicas malignas pueden extrapolarse a pacientes con otras enfermedades como la EPOC $2,7,33,38,46,47$.

Otras exploraciones de laboratorio, como la reacción en cadena de la polimerasa (PCR) o la concentración plasmática de -D-glucano están en desarrollo. Resultados preliminares sugieren que la PCR tiene una buena sensibilidad y especificidad ${ }^{48}$. Sin embargo, no hay métodos estandarizados y validados, las definiciones de API varían en los distintos estudios y hay que ser precavidos porque productos del plasma podrían inhibir los test de $\mathrm{PCR}^{49}$. La determinación de la concentración plasmática de -D-glucano, un componente de la pared celular del hongo, no parece superior a los test de PCR ${ }^{50}$.

Por otra parte, la producción de anticuerpos traduce la respuesta de la inmunidad humoral.
Constituyen un criterio diagnóstico en otras formas de enfermedad por Aspergillus, aunque su papel no está clarificado en la $\mathrm{API}^{7}$.

\section{Radiología}

La radiografía de tórax puede ser normal en los estadios iniciales de la API. Se han descrito múltiples signos radiológicos asociados con API, tales como consolidación, nódulos, el signo del halo o el signo del aire creciente. Lo más frecuente es la presentación de consolidación pulmonar, que presentan alrededor de $60 \%$ de pacientes ${ }^{3,11}$.

En un contexto clínico compatible, la presencia de una o varias opacidades nodulares debe hacernos sospechar API (Figura 2).

El signo del halo corresponde a una zona hemorrágica periférica a una lesión nodular (Figura 3). Su valoración muestra gran variabilidad según las características basales del paciente ${ }^{51}$.

El signo del aire creciente es el resultado de tejido pulmonar infartado delimitado por un espacio lleno de aire ${ }^{52}$.

El signo del halo o el signo del aire creciente, aunque poco frecuentes, son casi patognomónicos de API en pacientes hematológicos; aunque parece que se observan con menos frecuencia en pacientes con EPOC y trasplantados de órganos sólidos donde consolidaciones inespecíficas son vistas con más frecuencia que los hallazgos específicos mencionados ${ }^{4,11,53}$.

Otra característica del signo del halo es que se hace menos frecuente con la evolución de la enfermedad. Así, se puede ver en $68 \%$ de pacientes a los 3 días de evolución, pero sólo en $22 \%$ en el séptimo día ${ }^{4,11}$.

La tomografía computarizada (TAC) parece especialmente importante en el diagnóstico de esta enfermedad. Se ha demostrado que permite anticipar la sospecha diagnóstica de API 4,7 días comparado con la radiografía simple de tórax; o 11,5 días si se compara con el B-D-glucano ${ }^{54}$. Incluso, en pacientes inmunodeprimidos, algunos estudios han hallado que el diagnóstico se puede establecer antes con TAC que con GM, aunque carecemos de datos comparativos en pacientes con EPOC, donde la reactividad podría ser distinta ${ }^{38}$.

La supervivencia puede mejorar cuando se realiza una TC torácica al inicio de la enfermedad porque es bastante más sensible para detectar tem- 


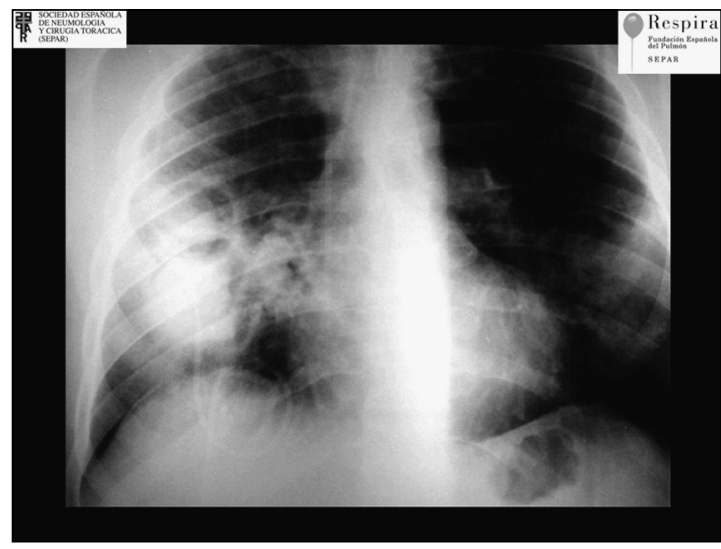

Figura 2. Radiografía de tórax de paciente inmunodeprimido con aspergilosis invasiva.

pranamente lesiones, que la radiografía de tórax ${ }^{55}$.

Además, la TAC puede ser útil en el diagnóstico diferencial, donde se incluyen otras infecciones o procesos no infecciosos como linfomas, neoplasias o tromboembolismo pulmonar ${ }^{56}$.

\section{Biopsia}

Como se ha dicho, solamente la histología puede confirmar la API junto a la presencia de otros criterios microbiológicos ${ }^{2}$ (Tabla 1). Las muestras de tejido pueden obtenerse mediante broncoscopia, biopsia percutánea o biopsia pulmonar abierta. Cualquiera de estas técnicas, dada la gravedad basal del paciente con EPOC y sospecha de API, plantean dificultades para su realización ${ }^{4}$.

Durante la fibrobroncoscopia, pueden estar presentes algunas lesiones en la mucosa que se pueden biopsiar. La rentabilidad de estas biopsias fue de $47 \%$ en pacientes con API probable o probada $^{57,58}$.

La biopsia transbronquial tiene una sensibilidad en torno $25 \%{ }^{36,57}$, pero es altamente específica, como parece demostrarse en un trabajo reciente, donde se demostraron Aspergillus invadiendo el parénquima pulmonar en todos los casos en que fue realizada, aunque los resultados deben ser tomados con cautela, ya que sólo se había realizado esta técnica en 5 pacientes ${ }^{3}$.

El tejido pulmonar puede ser obtenido también por biopsia percutánea con una rentabilidad de aproximadamente $50 \%$, si bien el resultado nega-

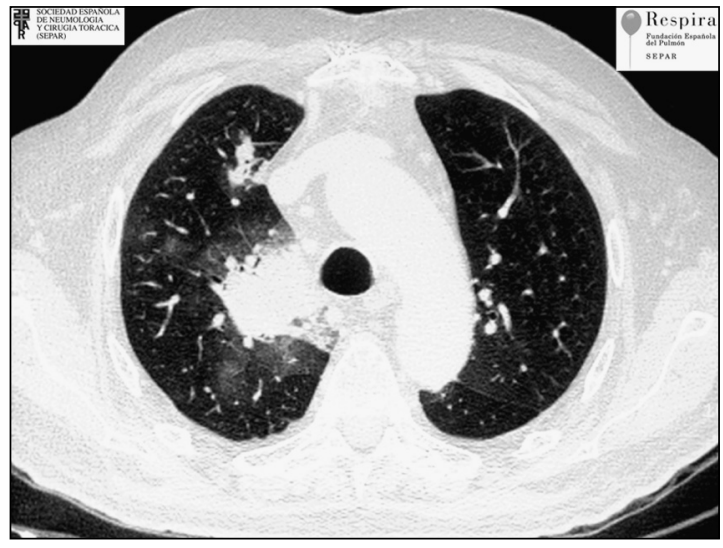

Figura 3. Nódulos y masas pulmonares bilaterales con halo periférico en vidrio deslustrado.

tivo no excluye el diagnóstico ${ }^{32}$. Esta técnica está especialmente indicada en lesiones periféricas, aunque el riesgo de neumotórax es importante en pacientes con $\mathrm{EPOC}^{59}$.

Además, la resección pulmonar puede ser también una herramienta terapéutica ${ }^{14}$.

\section{Conclusiones}

Aunque la colonización por Aspergillus spp es frecuente en los pacientes con EPOC, la API puede afectar a estos enfermos por lo que es muy importante establecer el diagnóstico lo antes posible para conseguir el mayor éxito terapéutico.

Debe sospecharse API en el paciente EPOC con una infección respiratoria resistente a los antibióticos y aspecto radiológico sugerente. En estos casos debe realizarse estudio de secreciones respiratorias, de los antígenos de Aspergillus y una TAC torácica. Este último estudio nos permitirá observar pequeños cambios en el parénquima pulmonar, anticipando la sospecha diagnóstica.

No hay suficientes datos para apoyar el uso de test serológicos (detección de galactomanano y anticuerpos) para establecer el diagnóstico, aunque los datos disponibles parecen prometedores.

Si el estado del paciente es grave, debería de iniciarse el tratamiento de forma empírica para proporcionarle mayores posibilidades de curación $\mathrm{y}$, posteriormente, deben de realizarse los procedimientos diagnósticos necesarios para confirmar la sospecha clínica de API. 
Los criterios diagnósticos establecidos para pacientes neutropénicos no son aplicables de forma definitiva en pacientes con otras características.

El diagnóstico definitivo requiere la confirmación histológica junto a la presencia de otros criterios microbiológicos o serológicos.

A los pacientes que reciban tratamiento crónico con corticoides, se les debería de proporcionar información acerca de cómo prevenir el contacto con fuentes medioambientales de Aspergillus spp.

Se necesitan llevar a cabo más trabajos para mejorar el conocimiento de la infección por Aspergillus en pacientes con EPOC y poder disminuir así la mortalidad de esta devastadora enfermedad.

Agradecimientos: Imágenes cedidas por el Fondo Iconográfico SEPAR (www.separ.es).

\section{Referencias}

1. Latgé JP. The pathobiology of Aspergillus fumigatus. Trends Microbiol 2001; 9: 382-9.

2. Bulpa P, Dive A, Sibille Y. Invasive pulmonary aspergillosis in patients with chronic obstructive pulmonary disease. Eur Respir J 2007; 30: 782-800.

3. Samarakoon P, Soubani AO. Invasive pulmonary aspergillosis in patients with COPD: a report of five cases and systematic review of the literature. Chron Respir Dis 2008; 5: 19-27.

4. Trof RJ, Beishuizen A, Debets-Ossenkopp YJ, Girbes AR, Groeneveld AB. Management of invasive pulmonary aspergillosis in non-neutropenic critically ill patients. Intensive Care Med 2007; 33: 1694-703.

5. Polat G, Urpek G, Yilmaz U, Büyüksirin M, Karada Polat $S$, et al. Successful treatment of invasive pulmonary aspergillosis in an immunocompetent host. Respirology 2005; 10: 393-5.

6. Lin SJ, Schranz J, Teutsch SM. Aspergillosis case-fatality rate: systematic review of the literature. Clin Infect Dis 2001; 32: 358-66.

7. Gangneux JP, Camus C, Philippe B. Epidemiology of and risk factors for invasive aspergillosis in nonneutropenic patients. Rev Mal Respir 2008; 25: 139-53.

8. Garnacho-Montero J, Amaya-Villar R. A validated clinical approach for the management of aspergillosis in critically ill patients: ready, steady, go! Crit Care 2006; 10: 132 .

9. Meersseman W, Vandecasteele SJ, Wilmer A, Verbeken E, Peetermans WE, Van Wijngaerden E. Invasive aspergillosis in critically ill patients without malignancy. Am J Respir Crit Care Med 2004; 170: 621-5.
10. Ascioglu S, Rex JH, de Pauw B, Bennett JE, Bille J, Crokaert F, et al. Defining opportunistic invasive fungal infections in immunocompromised patients with cancer and hematopoietic stem cell transplants: an international consensus. Clin Infect Dis 2002; 34: 7-14.

11. Maschmeyer G, Haas A, Cornely OA. Invasive aspergillosis: epidemiology, diagnosis and management in immunocompromised patients. Drugs 2007; 67: 1567-601.

12. Stergiopoulou T, Meletiadis J, Roilides E, Kleiner DE, Schaufele R, Roden M, et al. Host-dependent patterns of tissue injury in invasive pulmonary aspergillosis. Am J Clin Pathol 2007;127: 349-55.

13. Khasawneh F, Mohamad T, Moughrabieh MK, Lai Z, Ager J, Soubani AO. Isolation of Aspergillus in critically ill patients: a potential marker of poor outcome. J Crit Care 2006; 21: 322-7.

14. Garnacho-Montero J, Amaya-Villar R, Ortiz-Leyba C, León C, Álvarez-Lerma F, Nolla-Salas J, et al. Isolation of Aspergillus spp from the respiratory tract in critically ill patients: risk factors, clinical presentation and outcome. Crit Care 2005; 9: R191-9.

15. Kuwano K. Epithelial cell apoptosis and lung remodeling. Cell Mol Immunol 2007; 4: 419-29.

16. Latgé JP. Aspergillus fumigatus and Aspergillosis. Clin Microbiol Rev 1999; 12: 310-50.

17. Ng TTT, Robson GD, Denning DW. Hydrocortisoneenhanced growth of Aspergillus spp: implications for pathogenesis. Microbiology 1994; 140: 2475-9.

18. Philippe B, Ibrahim-Granet O, Prévost MC, GougerotPocidalo MA, Sánchez Pérez M, Van der Meeren A, et al. Killing of Aspergillus fumigatus by alveolar macrophages is mediated by reactive oxidant intermediates. Infect Immun 2003; 71: 3034-42.

19. Tuckermann JP, Kleiman A, Moriggl R, Spanbroek R, Neumann A, Illing A, et al. Macrophages and neutrophils are the targets for immune suppression by glucocorticoids in contact allergy. J Clin Invest 2007; 117: 1381-90.

20. Agarwal SK, Marshall GD Jr. Dexamethasone promotes type 2 cytokine production primarily through inhibition of type 1 cytokines. J Interferon Cytokine Res 2001; 21: 147-55.

21. Stuck AE, Minder CE, Frey FJ. Risk of infectious complications in patients taking glucocorticosteroids. Rev Infect Dis 1989; 11: 954-63.

22. Hartemink KJ, Paul MA, Spijkstra JJ, Girbes AR, Polderman $\mathrm{KH}$. Immunoparalysis as a cause for invasive aspergillosis? Intensive Care Med 2003; 29: 2068-71.

23. Ward NS, Casserly B, Ayala A. The compensatory antiinflammatory response syndrome (CARS) in critically ill patients. Clin Chest Med 2008; 29: 617-25. 
24. Saijo T, Izumikawa K, Takazono T, Kosai K, Kurihara S, Nakamura S, et al. A case of Legionella pneumophila pneumonia followed by invasive aspergillosis. Jpn J Infect Dis 2008; 61: 379-81.

25. Hasejima N, Yamato K, Takezawa S, Kobayashi H, Kadoyama C. Invasive pulmonary aspergillosis associated with influenza B. Respirology 2005; 10: 116-9.

26. Kishor U, Madan T, Sarma PU, Singh M, Urban BC, Reid KB. Protective roles of pulmonary surfactant proteins, SP-A and SP-D, against lung allergy and infection caused by Aspergillus fumigatus. Immunobiology 2002; 205: 610-8.

27. Madan T, Kishore U, Singh M, Strong P, Hussain EM, Reid KB, et al. Protective role of lung surfactant protein $\mathrm{D}$ in a murine model of invasive pulmonary aspergillosis. Infect Immun 2001; 69: 2728-31.

28. Madan T. Potential of lung surfactant proteins, SP-A and SP-D, and Mannan binding lectin for therapy and genetic predisposition to allergic and invasive aspergillosis. Recent Pat Inflamm Allergy Drug Discov 2007; 1: 183-7.

29. Andreas S, Heindl S, Wattky C, Möller K, Rüchel R. Diagnosis of pulmonary aspergillosis using optical brighteners. Eur Respir J 2000; 15: 407-11.

30. Greub G, Bille J. Aspergillus species isolated from clinical specimens: suggested clinical and microbiological criteria to determine significance. Clin Microbiol Infect 1998; 4: 710-716.

31. Verweij PE, Mellado E, Melchers WJ. Multiple-triazoleresistant aspergillosis. N Engl J Med 2007; 356: 1481-3.

32. Jantunen E, Piilonen A, Volin L, Parkkali T, KoukilaKähkölä P, Ruutu T, Diagnostic aspects of invasive Aspergillus infections in allogeneic BMT recipients. Bone Marrow Transplant 2000; 25: 867-71.

33. Caillot D, Mannone L, Cuisenier B, Couaillier JF. Role of early diagnosis and aggressive surgery in the management of invasive pulmonary aspergillosis in neutropenic patients. Clin Microbiol Infect 2001; 7 Suppl 2: 54-61.

34. Reichenberger F, Habicht J, Matt P, Frei R, Solèr M, Bolliger CT, et al. Diagnostic yield of bronchoscopy in histologically proven invasive pulmonary aspergillosis. Bone Marrow Transplant 1999; 24: 1195-9.

35. Brown MJ, Worthy SA, Flint JD, Müller NL. Invasive aspergillosis in the immunocompromised host: utility of computed tomography and bronchoalveolar lavage. Clin Radiol 1998; 53: 255-7.

36. Kahn FW, Jones JM, England DM. The role of bronchoalveolar lavage in the diagnosis of invasive pulmonary aspergillosis. Am J Clin Pathol 1986; 86: 518-23.

37. Maertens J, Van Eldere J, Verhaegen J, Verbeken E, Verschakelen J, Boogaerts M. Use of circulating ga- lactomannan screening for early diagnosis of invasive aspergillosis in allogeneic stem cell transplant recipients. J Infect Dis 2002; 186: 1297-306.

38. Weisser M, Rausch C, Droll A, Simcock M, Sendi P, Steffen I, et al. Galactomannan does not precede major signs on a pulmonary computerized tomographic scan suggestive of invasive aspergillosis in patients with hematological malignancies. Clin Infect Dis 2005; 41: 1143-9.

39. Meersseman W, Lagrou K, Maertens J, Wilmer A, Hermans G, Vanderschueren S, et al. Galactomannan in bronchoalveolar lavage fluid: a tool for diagnosing aspergillosis in intensive care unit patients. Am J Respir Crit Care Med 2008; 177: 27-34.

40. Nguyen MH, Jaber R, Leather HL, Wingard JR, Staley B, Wheat LJ, et al. Use of bronchoalveolar lavage to detect galactomannan for diagnosis of pulmonary aspergillosis among nonimmunocompromised hosts. J Clin Microbiol 2007; 45: 2787-92.

41. Pfeiffer CD, Fine JP, Safdar N. Diagnosis of invasive aspergillosis using a galactomannan assay: a meta-analysis. Clin Infect Dis 2006; 42: 1417-27.

42. Herbrecht R, Letscher-Bru V, Oprea C, Lioure B, Waller J, Campos F, et al. Aspergillus galactomannan detection in the diagnosis of invasive aspergillosis in cancer patients. J Clin Oncol 2002; 20: 1898-906.

43. Mennink-Kersten MA, Ruegebrink D, Klont RR, Warris A, Blijlevens NM, Donnelly JP, et al. Improved detection of circulating Aspergillus antigen by use of a modified pretreatment procedure. J Clin Microbiol 2008; 46: 1391-7.

44. Boutboul F, Alberti C, Leblanc T, Sulahian A, Gluckman E, Derouin F, et al. Invasive aspergillosis in allogeneic stem cell transplant recipients: increasing antigenemia is associated with progressive disease. Clin Infect Dis 2002; 34: 939-43.

45. Caillot D, Couaillier JF, Bernard A, Casasnovas O, Denning DW, Mannone L, et al. Increasing volume and changing characteristics of invasive pulmonary aspergillosis on sequential thoracic computed tomography scans in patients with neutropenia. J Clin Oncol 2001; 19: 253-9.

46. Sulahian A, Touratier S, Ribaud P. False positive test for Aspergillus antigenemia related to concomitant administration of piperacillin and tazobactam. N Engl J Med 2003; 349: 2366-7.

47. Mennink-Kersten MA, Ruegebrink D, Klont RR, Warris A, Gavini F, Op den Camp HJ, et al. Bifidobacterial lipoglycan as a new cause for false-positive platelia Aspergillus enzyme-linked immunosorbent assay reactivity. J Clin Microbiol 2005; 43: 3925-31. 
La aspergilosis pulmonar invasiva en la enfermedad pulmonar obstructiva crónica - J. M. Valle et al

48. White PL, Linton CJ, Perry MD, Johnson EM, Barnes RA. The evolution and evaluation of a whole blood polymerase chain reaction assay for the detection of invasive aspergillosis in hematology patients in a routine clinical setting. Clin Infect Dis 2006; 42: 479-86.

49. García ME, Blanco JL, Caballero J, Gargallo-Viola D. Anticoagulants interfere with PCR used to diagnose invasive aspergillosis. J Clin Microbiol 2002; 40: 1567-8.

50. Pazos C, Pontón J, Del Palacio A. Contribution of (1->3)-beta-D-glucan chromogenic assay to diagnosis and therapeutic monitoring of invasive aspergillosis in neutropenic adult patients: a comparison with serial screening for circulating galactomannan. J Clin Microbiol 2005; 43: 299-305.

51. Kuhlman JE, Fishman EK, Siegelman SS. Invasive pulmonary aspergillosis in acute leukemia: characteristic findings on CT, the CT halo sign, and the role of CT in early diagnosis. Radiology 1985; 157: 611-4.

52. Kuhlman JE, Fishman EK, Burch PA, Karp JE, Zerhouni EA, Siegelman SS. CT of invasive pulmonary aspergillosis. AJR Am J Roentgenol 1988; 150: 1015-20.

53. White DA. Aspergillus pulmonary infections in transplant recipients. Clin Chest Med 2005; 26: 661-74.

54. Kami M, Tanaka Y, Kanda Y, Ogawa S, Masumoto T, Ohtomo K, et al. Computed tomographic scan of the chest, latex agglutination test and plasma (1AE3)-betaD-glucan assay in early diagnosis of invasive pulmonary aspergillosis: a prospective study of 215 patients. Haematologica 2000; 85: 745-52.

55. Caillot D, Casasnovas O, Bernard A, Couaillier JF, Durand $\mathrm{C}$, Cuisenier B, et al. Improved management of invasive pulmonary aspergillosis in neutropenic patients using early thoracic computed tomographic scan and surgery. J Clin Oncol 1997; 15: 139-47.

56. Fedullo PF, Tapson VF. Clinical practice. The evaluation of suspected pulmonary embolism. N Engl J Med 2003; 349: 1247-56.

57. Albelda SM, Talbot GH, Gerson SL, Miller WT, Cassileth PA. Role of fiberoptic bronchoscopy in the diagnosis of invasive pulmonary aspergillosis in patients with acute leukemia. Am J Med 1984; 76: 1027-34.

58. Barile M, Bulpa P, Nollevaux MC, Dive A, González M. Usefulness of flexible bronchoscopy in ICU patients suspected of invasive aspergillosis. VIII Congreso Panamericano e Ibérico de Medicina Crítica y Terapia Intensiva. 17-21 de noviembre de 2007. Punta del EsteUruguay.

59. Denning DW. Diagnosis and management of invasive aspergillosis. Curr Clin Top Infect Dis 1996; 16: 277 99. 